\title{
Teaching and Learning Mode Reform in Digital Image Processing by Using Color Model
}

\author{
Tahamina Yesmin \\ Haldia Institute of Management, Haldia, West Bengal, India
}

\begin{abstract}
Article Info

Volume 7, Issue 4

Page Number : 10-16

Publication Issue :

July-August-2021

\section{Article History}

Accepted : 25 June 2021

Published : 02 July 2021

The traditional teaching mode of the digital image processing, which is centered on teachers, textbooks and classrooms, hinders the requirements of the image technology development and impedes the cultivation of the innovative talents. Though the digital image processing is one of the emerging technologies, it has been rapidly developed and widely used in the electronic information technology. In order to adapt to its development, this paper has explored the teaching and practice reform on the digital image processing by different color mode and to improve the understanding of students as well as teacher by clustered image which will enhance the core idea of a particular image by exploring all the colors present in every part of image. Students can better gain their knowledge skill by observing those images in colored clustered form.

Keywords : Image Color Analysis, Digital Image Processing, Teaching and Learning mode, RGB Color Model, Image Segmentation.
\end{abstract}

\section{INTRODUCTION}

Pictures are the most common and convenient means of conveying or transmitting information. A picture is worth a thousand words. Pictures concisely convey information about positions, sizes and interrelationships between objects. They portray spatial information that we can recognize as objects. Human beings are good at deriving information from such images, because of our innate visual and mental abilities. About $75 \%$ of the information received by human is in pictorial form. Teachers have tried to teach their students by introducing text books along with verbal instructions in traditional education system. However, teaching and learning methods could be changed for developing Information and Communication Technology (ICT). It's time to adapt students with interactive learning system so that they can improve their learning, catching, and memorizing capabilities. It is indispensable to create high quality and realistic leaning environment for students.

Image is an important form of conveying teaching information and carrying educational content. In the teaching process, a variety of media information integrating text, graphics/images, audio and even video technologies can stimulate the learner's interest in learning and improve the quality of teaching. In distance education, the greatest advantage of images is their figurativeness. Many of the contents of teaching will appear "dry" and boring if they are only expressed in words, and the expression will become very "rich and colorful" with images. In the so-called 
a picture wins thousands of words, image teaching is conducive to fully activate the classroom learning atmosphere and inject vitality into teaching [1].

In many applications such as remote sensing, medical imaging, security, computer vision, multi-camera video and panoramic image, correcting nonlinear distortion caused by optical lens has always been a hot research topic [2]. The distortion correction of the image can be generally started from two aspects, optical design correction and digital image processing correction. The use of optical and mechanical structures for lens correction has many limitations in terms of design difficulty, manufacturing, and lens size and cost. Therefore, correction using digital image processing has become the mainstream of society. The use of digital image processing technology to achieve image correction of the camera is usually divided into two steps, camera calibration and image correction [3].

ICT as a lever for educational change is influenced by the teacher who has to implement technology in the classroom and can implement technology for different purposes (as a basic ICT-skills tool, a learning tool, an information tool). Followed by the actual use of the teacher, ICT-related teacher conditions are essential, such as teacher competence to deal with the technology and professional development. Next, ICT-related school conditions are needed; such as ICT-support, ICT-coordinators and ICT infrastructure. Finally, school improvement conditions such as leadership, and the relation between teachers, and participative-decision making are relevant [4].

Visual learning can be easier to understand and deal with their learning. We developed visual learning materials (an overview of solar system) in the form of video for students of primary level using different multimedia application tools. The objective of this paper is to examine the impact of student's abilities to acquire new knowledge or skills through visual learning materials and blended leaning that is integration of visual learning materials with teacher's instructions.

For a true color composite an image data used in red, green and blue spectral region must be assigned bits of red, green and blue image processor frame buffer memory. A color infrared composite 'standard false color composite' is displayed by placing the infrared, red, green in the red, green and blue frame buffer memory.

Image segmentation is partitioning of an image into segments on the basis of homogeneous features, similarity between pixels in a specified region depends on different criteria like color, intensity or texture, so that objects in an image can be identified based on variations of intensity in an image. This paper gives an overview of the color models by stating their advantages and problems found when they are implemented.

Image segmentation is one of the mostly used methods to classify the pixels of an image correctly in a decision-oriented application. It divides an image into a number of discrete regions such that the pixels have high similarity in each region and high contrast between regions. It is a valuable tool in many fields including health care, image processing, traffic image, pattern recognition etc. There are different techniques for image segmentation like threshold based, edge based, cluster based, and neural network based. From the different technique one of the most efficient methods is the clustering method. Again, there are different types of clustering: K-means clustering, Fuzzy C-means clustering, mountain clustering method and subtractive clustering method. One of most used clustering algorithms is k-means clustering. It is simple and computationally faster 
than the hierarchical clustering. And it can also work for large number of variables. But it produces different cluster result for different number of numbers of cluster. So, it is required to initialize the proper number of numbers of cluster, K2. Again, it is required to initialize the $\mathrm{k}$ number of centroids. Different value of initial centroid would result different cluster. So, selection of proper initial centroid is also an important task.

\section{Literature Review}

Ibrahim A. Almerhag, Idris S Feghi and Ali A Dulla [5] proposed a new method of $\mathrm{k}$-means clustering algorithm in," A modified k-means clustering algorithm for gray image segmentation". It proposed a new modified method of conventional k-means algorithm. In conventional k-means algorithm, usually cluster centers are randomly initialized. But here it intruded a new method to initialize the initial cluster centers. It uses minimum and maximum data points in the given data set to initialize the cluster centers. Then comparison is done between the standard and proposed k-means algorithm and it is found that the proposed algorithm has effective and more robust than the traditional $\mathrm{k}$-means algorithm.

Alan Jose, S. Ravi and M. Sambath proposed Brain Tumor Segmentation using K-means Clustering and Fuzzy C-means Algorithm and its area calculation. In the paper, they divide the process into three parts, pre-processing of the image, advanced k-means and fuzzy c-means and lastly the feature extraction. First pre-processing is implemented by using the filter where it improves the quality of the image. Then the proposed advance $\mathrm{K}$-means algorithm is used, followed by Fuzzy c-means to cluster the image. Then the resulted segment image is used for the feature extraction for the region of interest. They used MRI image for the analysis and calculate the size of the extracted tumor region in the image [6].
Khaled Hammouda has done a survey on different techniques of data clustering in, "A comparative study of data clustering technique". It implemented different data clustering technique- k-means clustering, Fuzzy c-means clustering, and Mountain clustering and subtractive clustering. It analyzes this different technique, it is found that $\mathrm{k}$-means and Fuzzy c-means are preferred when the number of clusters is known, and in such cases Mountain clustering is not usually used because of high number of dimension due to its exponential proportionality to the dimension of the problem. But if the number of clusters is not known, Mountain clustering method is used. Again, it is concluded that subtractive clustering is more advantages than Mountain clustering. Lastly it is stated that this different technique can be used in conjunction with other neural or fuzzy system so that it can improve the system performance [7].

Madhu Yedla, Srinivasa Rao Pathakota, T. M. Srinivasa6 proposed Enhancing K-means clustering algorithm with improved initial center. A new method for finding the initial centroid is introduced and it provides an effective way of assigning the data points to suitable clusters with reduced time complexity. They proved their proposed algorithm has more accuracy with less computational time comparatively original k-means clustering algorithm. This algorithm does not require any additional input like threshold value. But this algorithm still initializes the number of cluster $\mathrm{k}$ and suggested determination of value of $\mathrm{k}$ as one of the future works [8].

Gudrun J. Klinker, Steven A. Shafer and Takeo Kanade observed Physical Approach to Color Image understanding with additional example through opposed to $\mathrm{k}$ means and Anil Z Chitade evaluated the Color based image segmentation using k-means clustering. R. C. Gonzalez and R. E. Woods described Digital Image Processing where YZang presented a 
review on image segmentation techniques with remote sensing perspective. [9].

Y. Li and Y. Shen done a great job about Robust image segmentation algorithm using fuzzy clustering based on kernel-induced distance measure whereas D. E. Ilea and P. F. Whelan gave a complete overview about Color image segmentation using a spatial kmeans clustering algorithm [10].

Though the existing curriculum system of electronic information has been set up relatively perfectly and the practice of specialized course have been established comparatively comprehensively, the current curriculum framework is played attention to the knowledge teaching and ignored the undergraduate's ability training of introducing, analyzing, solving and evaluating question. Therefore, it is lack of the ability training that the students' technology innovation abilities are restricted. Then, the reform of teaching practice mode of digital image processing curriculum is not only feasible, but also very necessary [11]. [12], [13].

Modern education and communication environments can offer alternative ways in the learning process. Technology has been widely used in educational technologies. It is very near when multimedia tools will be perfect utilization in education sector. Using interactive multimedia in the teaching process is growing in the present context. Interactive learning plays a very important role in assisting students in learning processes [14].

It has possibility to enhance the early education system with multimedia technologies. The positive impact of the developed program on student's abilities is to understand new knowledge or skills. Multimedia education offers an alternative to traditional education that can enhance the current methods and provide an alternative.
There have been many works done in the area of image segmentation by using different methods. And many are done based on different application of image segmentation. Many methods of image preprocessing techniques had been already existed. But with increase in the usage of image processing nowadays many are trying to produce better and new efficient method to meet the requirement different application. K-means algorithm is the one of the simplest clustering algorithms and there are many methods implemented so far with different method. And many researchers are also trying to produce new methods which are more efficient than the existing methods, and shows better segmented result. Some of the existing recent works are discussed here.

\section{Research Process}

The algorithm consists of $\mathrm{k}$-means clustering, partial contrast stretching and median filter. Mostly the medical images which are used for segmentation have low contrast but, in this paper, I am trying to apply these methods for study related image and videos also. So, contrast stretching is used to improve the quality of the image. After improving the quality of image, subtractive clustering algorithm is used to generate the centers, based on the potential value of the image. Number of centers is generated based on number of cluster $\mathrm{k}$. This center is used as initial center in $\mathrm{k}$ means algorithm. Using the k-means algorithm, the image is segmented into $\mathrm{k}$ number of clusters. After the segmentation of image, the image can still contain some unwanted region or noise. These noises are removed by using the median filter. Following figure 1: is the process how this algorithm will work. 


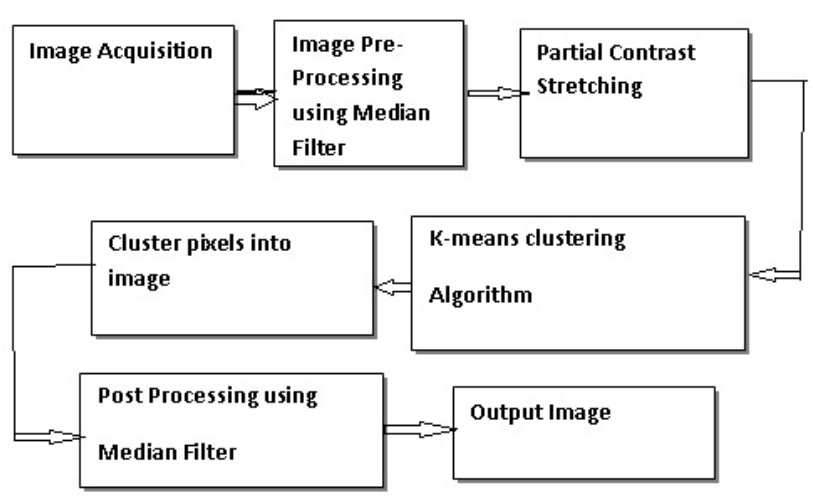

Figure 1

\section{Image Acquisition}

The general aim of Image Acquisition is to transform an optical image (Real World Data) into an array of numerical data which could be later manipulated on a computer, before any video or image processing can commence an image must be captured by camera and converted into a manageable entity. The Image Acquisition process consists of three steps: -

1. Optical system which focuses the energy

2. Energy reflected from the object of interest

3. A sensor which measure the amount of energy.

\section{Partial Contrast Stretching:}

Medical images which have been used for the analysis may have their own weakness such as blurred or low contrast. So, a contrast enhancement technique such as Partial Spatial Starching (PCS) is used to improve the image quality and contrast of the image8. It is done by stretching and compression process. By applying this technique, the pixel range of lower threshold value and upper threshold value will be mapped to a new pixel range and stretched linearly to a wide range of pixels within new lower stretching value, and the remaining pixels will experience compression.

\section{Median Filter:}

Median filtering is used as a noise removal in order to obtain a noise free image. After segmentation is done, the segmented image may still present some unwanted regions or noise. So, to make the image a good and better quality, the median filter is applied to the segmented image. We can use different neighborhood of $\mathrm{n} \times \mathrm{n}$. But generally, neighborhood of $\mathrm{n}=7$ is used because large neighborhoods produce more severe smoothing. The median filter is a nonlinear signal processing technology based on statistics. The noisy value of the digital image or the sequence is replaced by the median value of the neighborhood (mask). The pixels of the mask are ranked in the order of their gray levels, and the median value of the group is stored to replace the noisy value.

\section{K-Means Clustering Algorithm:}

Clustering is a method to divide a set of data into a specific number of groups. It's one of the popular methods is $\mathrm{k}$-means clustering. In $\mathrm{k}$-means clustering, it partitions a collection of data into a $\mathrm{k}$ number group of data [15]. It classifies a given set of data into $\mathrm{k}$ number of disjoint clusters. K-means algorithm consists of two separate phases. In the first phase it calculates the $\mathrm{k}$ centroid and in the second phase it takes each point to the cluster which has nearest centroid from the respective data point. There are different methods to define the distance of the nearest centroid and one of the most used methods is Euclidean distance. Once the grouping is done it recalculate the new centroid of each cluster and based on that centroid, a new Euclidean distance is calculated between each center and each data point and assigns the points in the cluster which have minimum Euclidean distance. Each cluster in the partition is defined by its member objects and by its centroid. The centroid for each cluster is the point to which the sum of distances from all the objects in that cluster is minimized. So, $\mathrm{K}$-means is an iterative 
algorithm in which it minimizes the sum of distances from each object to its cluster centroid, over all clusters.

Let us consider an image with resolution of $\mathrm{x} \times \mathrm{y}$ and the image have to be cluster into $k$ number of clusters. Let $\mathrm{p}^{\mathrm{i}}(\mathrm{x}, \mathrm{y})$ be an input pixel to be cluster for each $R, G, B$ channel. The algorithm for $k$-means clustering is following as:

\section{Load color image.}

2. Separate the image into red, green, blue channel.

3. Initialize number of cluster $\mathrm{k}$ and center for each channel.

4. For each pixel of an image, calculate the Euclidean distance $d$, between the center and each pixel of an image using the relation given below. i represent each channel and $\mathrm{k}$ represent particular cluster.

5. Assign all the pixels to the nearest center based on distance d.

6. After all pixels have been assigned, recalculate new position of the center of each channel by averaging the pixels.

7. Repeat the process until it satisfies the tolerance or error value.

8. Reshape the cluster pixels into image.

Although k-means has the great advantage of being easy to implement, it has some drawbacks. The quality of the final clustering results is depending on the arbitrary selection of initial centroid. So, if the initial centroid is randomly chosen, it will get different result for different initial centers. So, the initial center will be carefully chosen so that we get our desire segmentation. And also, computational complexity is another term which we need to consider while designing the $\mathrm{K}$-means clustering. It relies on the number of data elements, number of clusters and number of iterations.

Here I have applied K-means clustering method to show the difference between original and clustered image for betterment of teaching and learning method clearer to understand well by using image.
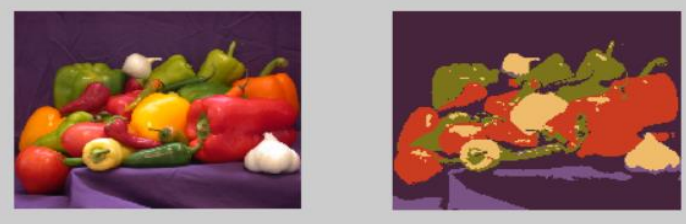

Figure 2 : Image using K-means Clustering Algorithms

\section{CONCLUSION}

The characteristics of digital image processing course are analyzed in this paper, which reflects the perfect combination of the theory and experiment teaching, the practice and innovation training. Therefore, the digital image curriculum should not only own the basic knowledge modules, but also embodies professional skill modules. In this paper I am trying to show that the improvement of students learning skills especially when interactive learning materials are used as the main resources by the teacher, although we have no intention the replacement of traditional education system. Just we wanted to introduce a method for promoting learning and quick adaptation with learning materials. This paper has explored the teaching and practice reform on the digital image processing curriculum, including the teaching objectives specially with the help of Kmeans clustering algorithms. By using algorithms on image, we can ger more observed value and color 
cluster system in a particular image. In teaching and learning process if color clustered can input we can get more idea about many colored mage processes.

\section{REFERENCES}

[1] Y. Xu, J.T. Dong, Z.Q. Wang, 'Fuzzy based distance correction algorithm for digital image interpolation', Appl. Mech. Mater. 513-517(513 517), 1549-1554 (2014).

[2] Y. Zhu, B. Chen, M. Qin, et al., 2-D micromachined thermal wind sensors-a review. IEEE Internet Things J. 1(3), 216-232 (2017).

[3] S.W. Zhang, X.N. Zhang, Z.Y. Wu, et al., Research on asphalt mixture injury digital image based on enhancement and segmentation processing technology. Appl. Mech. Mater. 470, 832-837 (2014).

[4] Vanderlinde R, van Braak J. The e-capacity of primary schools: Development of a conceptual model and scale construction from a school improvement perspective. Computers \& Education. 2010; 55, 541-553.

[5] Ibrahim A. Almerhag, Idris S. E, Feighi and Ali A Dulla, "Modified k-means clustering algorithm for gray image segmentation", Naseer International University, Tarhoona, Libya.

[6] Alan Jose, S. Ravi and M. Sambath, Brain Tumor Segmentation using K-means clustering and Fuzzy C-means Algorithm and its Area Calculation. In International Journal of Innovative Research in Computer and Communication Engineering, vol. 2, issue 2, March (2014).

[7] Khali Hamuda, "A Comparative study of data clustering technique", Department of system Design Engineering, University of Waterloo, Canada.

[8] Madhu Yedla, Srinivasa Rao Pathakota and T. M. Srinivasa, Enhanced K-means Clustering Algorithm with Improved Initial Center, In International Journal of Science and Information Technologies, vol. 1(2), pp. 121-125, (2010).
[9] Gudrun J. Klinker, Steven A. Shafer and Takeo Kanade, "A Physical Approach to Color Image Understanding," International Journal of Computer Vision, Vol. 4, No. 1, pp. 738, 1990.

[10] Y. Li and Y. Shen, "Robust image segmentation algorithm using fuzzy clustering based on kernelinduced distance measure," in Proceedings of the International Conference on Computer Science and Software Engineering, 2008, vol. 1, pp. 1065-1068.

[11] Aimi Salihai, Abdul Yusuff Masor, Zeehaida Mohamed, " Color image segmentation approach for detection of Malaria parasites using various color models and k-means clustering", in Wseas Transaction on Biology and Biomedicine, vol. 10, January 2013.

[12] C. Yan et al., "Teaching system of toilet spray glaze based on digital image processing," International Journal of Digital Content Technology \& Its Application, vol. 7, no. 9, p. 215, August 2013.

[13] A. G. D. Asir and L. E. Jebamalar, "Competency based calisthenics of learning outcomes for engineering education," International Journal of Education and Learning, vol. 2, no. 1, pp.25-34, May 2013.

[14] S. Nusir, I. Alsmadi, M. Al- kabi, and F. Shardqah, (2011) "Designing an Interactive Multimedia Learning System for the Children of Primary Schools in Jordan", IEEE Global Engineering Education Conference (EDUCON), April 4-6, Amman, Jordan.

[15] Sorin Israil," An overview of clustering methods", in With Application to Bioinformatics.

\section{Cite this article as :}

Tahamina Yesmin, "Teaching and Learning Mode Reform in Digital Image Processing by Using Color Model", International Journal of Scientific Research in Computer Science, Engineering and Information Technology (IJSRCSEIT), ISSN : 2456-3307, Volume 7, Issue 4, pp.10-16, July-August-2021. Available at doi: https://doi.org/10.32628/CSEIT2173174 Journal URL : https://ijsrcseit.com/CSEIT2173174 